\title{
Takotsubo syndrome: consequence or cause of ischemic stroke
}

Dear Sir,

With interest we read the article by Bersano et al. (2014) about a 70-year-old female who is reported to have developed Takotsubo syndrome (TTS) after a left temporal lobe ischemic stroke. We have the following comments and concerns.

Although ischemic stroke has repeatedly been reported to cause TTS, it is not convincing in each of these cases that TTS was truly a consequence and not the cause of stroke. Thus, it would be interesting to know in the presented case whether TTS was truly a complication of the initial stroke or the cause of the first stroke. Did the patient report chest pain already on admission, was routine ECG on admission truly normal, and did she have dyspnea or leg edema on admission? The authors mention that the patient had mental stress and pneumonia prior to suddenonset aphasia, classical triggers of TTS, which could have triggered TTS in the presented patient. Continuous ECG monitoring on a stroke unit does not usually allow assessment of ST segment alterations, QT prolongation or Twave abnormalities. Were such changes present on the admission ECG or on routine ECGs recorded prior to the second stroke?

The authors do not report whether the patient had undergone coronary angiography, which is a prerequisite to diagnose TTS. CT angiography of the coronary arteries has the disadvantage of over-or under-estimating coronary heart disease and is not the gold standard. Why was conventional coronary angiography not carried out to exclude coronary heart disease?

Heparinization is contraindicated in the acute stage of ischemic stroke if there is a severe territorial infarct, as in the present case with an NIHSS score of 12. The authors do not present imaging of the first and second stroke. What was the size of these strokes? Were there any indications for a hemorrhagic transformation? How high was the risk of bleeding when starting heparin? Was intravenous heparin therapy complicated by intracerebral bleeding or hemorrhaghic transformation of the first or second stroke? Why did the patient not undergo systemic thrombolysis or mechanical thrombus extraction in the acute stage of the first stroke? Which branches of the median cerebral artery were occluded?

The fact that the intraventricular thrombus was not detected earlier than on the day of the second event does not exclude that it was already present on the day of admission. Did the patient receive $\beta$-blockers after detection of TTS?

Yoshimura et al. (2008) was not the first to describe stroke-induced TTS. Stroke-induced TTS was reported previously by Varela et al. (2006), by Abi-Saleh et al. (2006), and by Sánchez Flores (2005). Most likely, it was Wang et al. (1997) who first described stroke-induced TTS.

So far TTS has been reported as a complication of ischemic stroke in >20 cases of ischemic stroke (Finsterer et al., 2014). Concerning central nervous system disease as the trigger of TTS, ischemic stroke is the third most frequent trigger after subarachnoid bleeding and epilepsy (Finsterer et al., 2014).

Overall, this interesting case requires re-evaluation concerning the causative role of TTS. The earlier adequate treatment is applied to TTS patients the more likely it is that severe complications of TTS will be avoided.

\author{
Josef Finsterer, $M D, P h D^{a}{ }^{*}$, Rahim Aliyev, $M D^{b}$ \\ ${ }^{a}$ Krankenanstalt Rudolfstiftung, Vienna, Austria \\ ${ }^{b}$ Department of Neurology, Azerbaijan State Advanced Training Institute for Doctors named after A. Aliyev, \\ Baku, Azerbaijan \\ *Correspondence to: fifigs1@yahoo.de
}

\section{References}

Abi-Saleh B, Iskandar SB, Schoondyke JW, et al (2006). Tako-tsubo syndrome as a consequence of transient ischemic attack. Rev Cardiovasc Med 7:37-41.

Bersano A, Melchiorre P, Moschwitis G, et al (2014). Tako-tsubo syndrome as a consequence and cause of stroke. Funct Neurol 29:135-137. 
Finsterer J, Wahbi K (2014). CNS disease triggering Takotsubo stress cardiomyopathy. Int J Cardiol 177: 322-329.

Sánchez Flores M, Marcos Martín M, Cruz González I, et al (2005). Intraventricular thrombus associated with Tako-Tsubo syndrome in a patient with previous transient ischemic stroke. Med Clin (Barc) 125:237.

Varela D, Díaz F, Hlavnicka A, et al (2006). Stunned myocardium after acute ischemic stroke. Medicina (B Aires) 66:249-253.

Wang TD, Wu CC, Lee YT (1997). Myocardial stunning after cerebral infarction. Int J Cardiol 58:308-311. 